\title{
EMBARAZO FIMBRICO A TERMINO
}

\section{Presentación de 3 Casos}

Dra. Laura Rojas R.*

Dr. Jaime Cantillo G.**

Son muchas las publicaciones nacionales y extranjeras sobre embarazo extrauterino a término; a pesar de ellas, queremos hacer una contribución a la literatura presentando 3 nuevos casos, ocurridos en el Instituto Materno Infantil (IMI) de Bogotá, desde el VI-I-65 hasta la fecha (31-VII-71), por ser una entidad poco frecuente, en la cual hay que pensar, pues del diagnóstico oportuno depende la supervivencia fetal.

La primera referencia de embarazo abdominal primario la hizo Tait en 1888. En Colombia Pompilio Martínez (1) describe el primer caso en 1912, un tubárico a término. Roberto Serpa (2) obtiene por laparotomía el primer feto vivo masculino de 3.500 grs. en 1923. Guillermo Navas en su tesis de grado publica un caso de Jorge Solanilla en Cali con supervivencia materna y fetal (3). En nuestra Institución (IMI.) son varias las publicaciones al respecto: Ramírez Merchán (4), Gómez Palacino (5-6), Sánchez Torres (7) y Guzmán Alandette (8), pero ninguna relata supervivencia fetal.

Botero U. en Medellín (9) describe 8 casos con supervivencia fetal en 3 de ellos. Acosta B. (10) presenta 2 casos, uno tubo-intersticial $y$ el otro abdominal secundario. Londoño U. (11) describe un caso más en una mujer de 38 años con antecedentes de cesárea corporal. Gómez Peña (12) en Venezuela trató un caso de embarazo tubárico gemelar cuyos fetos pesaron 3.340 y 120 grs. cada uno.

Los embarazos abdominales primarios son muy pocos y requieren la integridad de los órganos pélvicos genitales. Friedrich y col. (13) en 1968, recopilaron 24 casos de la literatura mundial, sin que ninguno pasara de la 12a semana de gestación. Near y col. (14) relata un caso de implante en hígado con feto vivo de más o menos 22 semanas de gestación, en una mujer que fue intervenida con el diagnóstico de absceso hepático, falleciendo en el acto quirúrgico por shock hipovolémico debido a la profusa hemorragia que se produjo al liberar el saco amniótico donde estaba el feto.

Los embarazos ováricos representan una incidencia baja de los extra-

* Instructora Asociada de Ginecología y Obstetricia de la Facultad de Medicina de la Universidad Nacional de Colombia.

** Instructor Asociado de Anatomía Patológica de la Facultad de Medicina de la Universidad Nacional de Colombia. 
uterinos, correspondiéndoles del 0.06 al $0.94 \%$. Entre nosotros Ramírez y Merchán (4) en 1946 publicó 2 casos que llegaron casi a término, uno de los cuales se comprobó histológicamente. Hoang $\mathrm{Ngoc}$ (15) consigna un caso auténtico confirmado por anatomía patológica.

Dentro de la literatura hay relatos que causan curiosidad, como el de Hutchinson (16), quien presenta el caso de un embarazo tópico concomitante con un ectópico; seis años después la madre y sus dos hijos se encontraban en perfectas condiciones. Sehder (17) cuenta de una paciente negra de 22 años con embarazo gemelar de 34 semanas, a quien se le practica cesárea por distocia cervical, después de extraer el primer feto de 1.800 grs., encuentra el segundo gemelo de 1.100 grs. en el anexo derecho; este último murió por múltiples malformaciones congénitas.

Queremos llamar la atención: por ser una entidad poco frecuente, el diagnóstico no se hace, casi siempre es un hallazgo quirúrgico y lo más grave de todo, cuando el feto ha dejado de existir; en ocasiones es mucho más tardío cuando el feto sufre ya petrificación confundiéndose con quistes de ovario $(3,18$ y 33 ).

La anamnesis es muy importante porque permite obtener datos sobre complicaciones en los primeros meses del embarazo, operaciones previas, cesáreas, miomectomías o maniobras abortivas. El tacto vaginal es una buena ayuda para el diagnóstico porque el cuello y el cuerpo uterino no sufren las modificaciones del embarazo. La ausencia de las contracciones de Braxton Hicks y la falta de respuesta a los ocitócicos contribuyen a confirmar el diagnóstico; lo mismo, los Rx. de abdómen que muestran presentaciones anormales, especial- mente situación transversa alta; falta de contorno uterino alrededor del feto, y en la placa lateral, las partes fetales por detrás o montadas sobre la columna lumbar de la madre. La histerosalpingografía confirma el diagnóstico, (19, 20, 21). K'ey y Yen-Ling (22) estudian 12 casos, confirmando el diagnóstico con histerosalpingografía en 4 de ellos. Una vez hecho el diagnóstico la conducta es siempre quirúrgica con el fin de extraer el feto y la placenta, si es fácil su extracción; de lo contrario, es mejor dejarla in situ. Ella subinvoluciona presentando isquemia y cambios degenerativos dentro de las 6 a 10 semanas subsiguientes a la operación, hecho que se ha comprobado radiológicamente por estudios de flebografías y arteriogramas femorales $(23,24,25$ y 26$)$. En el $29 \%$ de los embarazos abdominales, hay que dejar la placenta por la imposibilidad de extraerla y por su alto riesgo de hemorragia profusa que puede desencadenar la muerte de la paciente.

\section{Material}

PRIMER CASO. H. Clínica No 196188. Paciente de 30 años ( 1 Po) que ingresa (28VIII-68) consultando por ausencia de movimientos fetales desde hacía 15 días. Asistió a consulta externa prenatal durante todo su embarazo y el feto permaneció vivo hasta la 39a. semana; el embarazo se prolonga y en la $43 a$. semana ya no siente al feto. Se hospitaliza con el diagnóstico de feto muerto $y$ se induce con pitocín en 4 ocasiones, sin lograr ningún resultado. Se valora el caso y se encuentra un cuello duro, central, cerrado. El útero se palpa rechazado a la izquierda por la masa de anexo derecho descrita como útero grávido. La histerectomía fue de 8 cms., la histerosalpingografía y los $\mathrm{Rx}$. de abdómen confir. man el diagnóstico de embarazo abdominal secundario. (Figs. Nos. 1 y 2). En el acto operatorio se aprecia masa quística dependiente de anexo derecho con adherencia a los órganos vecinos. (Fig. Nọ 3). Utero $y$ anexo izquierdo normales. Se le practica salpingooforectomía derecha y apendicectomía. (P. Q. N 218-69) de anatomía patológica. 


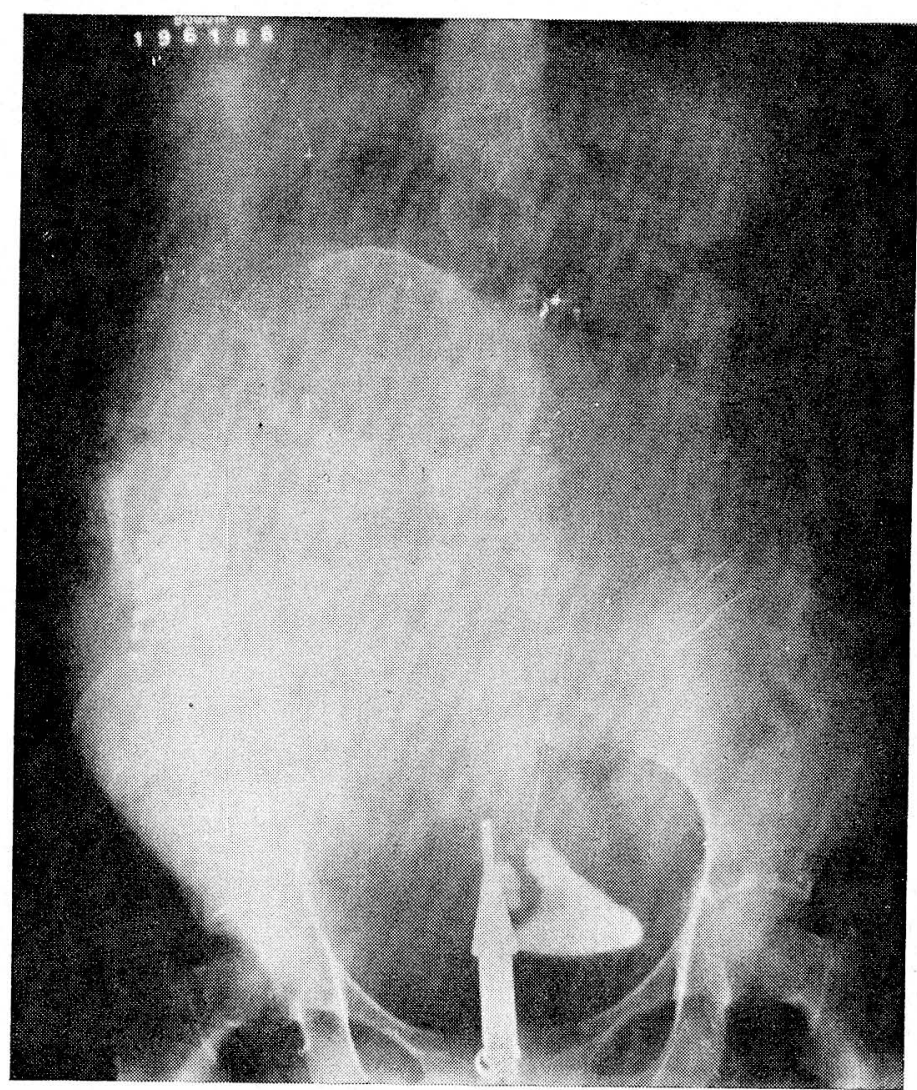

FIGURA No 1 - La histerosalpingografía del primer caso muestra el útero de tamaño normal y el feto al lado de él.

\section{Descripción macroscópica.}

El espécimen consiste en un saco amniótico que es ovalado y mide 30 × 28 × 25 cms., cuya superficie exterior presenta múltiples adherencias y numerosos vasos de mediano calibre. La trompa está adherida en uno de sus bordes $y$ es permeable en sus dos tercios internos; la fimbria y el tercio externo se observan severamente ensanchados tomando la forma de embudo que se confunde insensiblemente con la placenta donde tuvo implante el huevo. Al incidir el saco, sale un líquido turbio meconiado, de color oscuro y se visualiza un feto que mide $54 \mathrm{cms}$. de longitud $y$ pesa 2.850 grs., macerado, femenino y con signos de hipermadurez. No existe ningún tipo de malformación congénita. El feto en general es totalmente normal. Al disecar el saco se pueden ver el amnios, la decidua y el corion. Los múltiples cortes practicados a la placenta muestran varios infartos. El apéndice está adherido al saco amniótico con exudado de fibrina en la serosa. El ovario mide $3 \times 2 \mathrm{cms}$. y al corte presenta el cuerpo amarillo del embarazo (Fig. No 4).

\section{Descripción microscópica}

Los cortes practicados a nivel de la fimbrina muestran este órgano, donde existen vellosidades coriales con signos de degeneración. Histológicamente se comprueba con claridad que la placenta se implantó a nivel de la fimbria (Fig. No 5). El ovario es normal. Los vasos de la fimbria son bastante grandes sin signos de degeneración. 


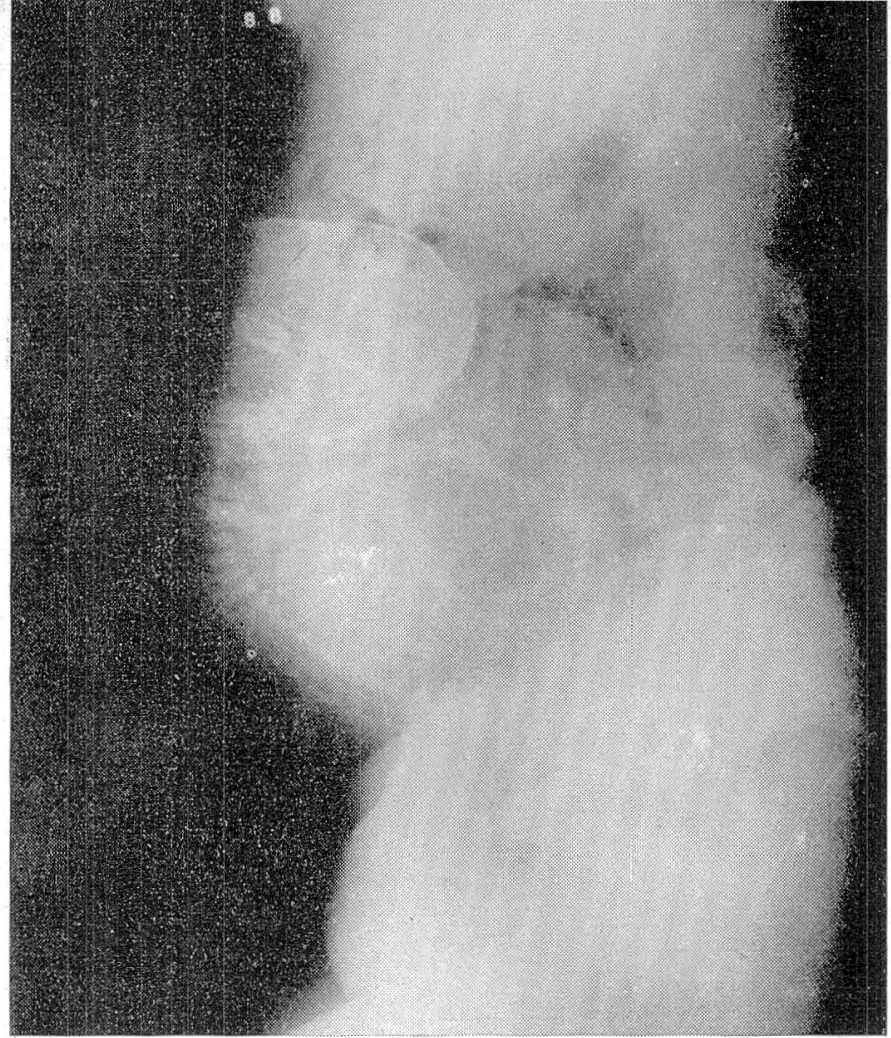

FIGURA No 2 - Rx. lateral del mismo caso.

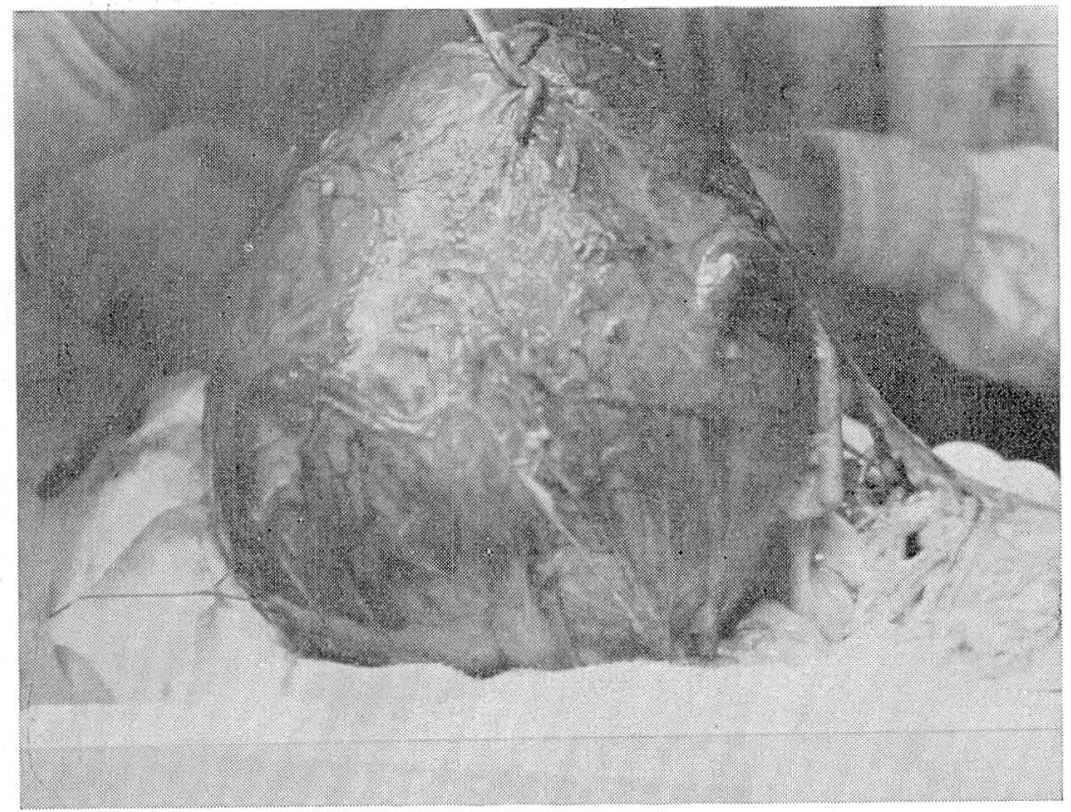

FIGURA № 3 - Aspecto del saco amniótico en el acto quirúrgico presentando múltiples adherencias. 


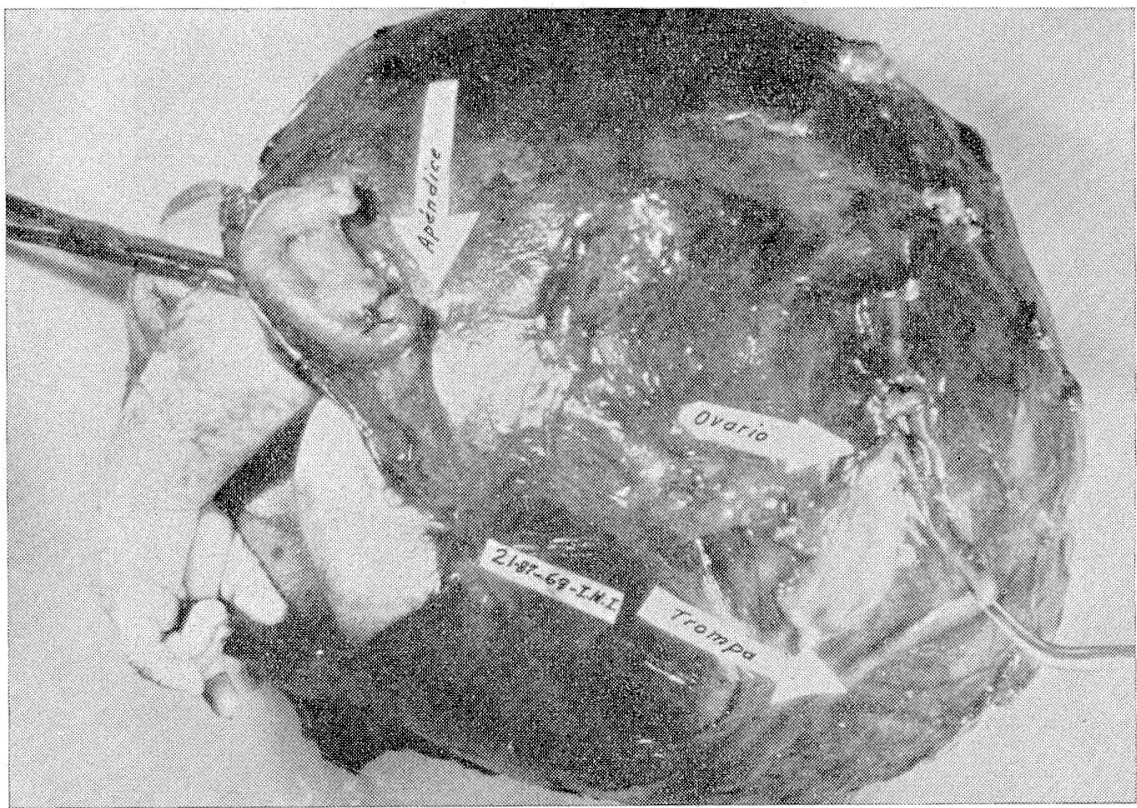

FIGURA No 4 - El espécimen quirúrgico que presenta el feto, la trompa, el ovario y el apéndice vermiforme adherida al saco amniótico.

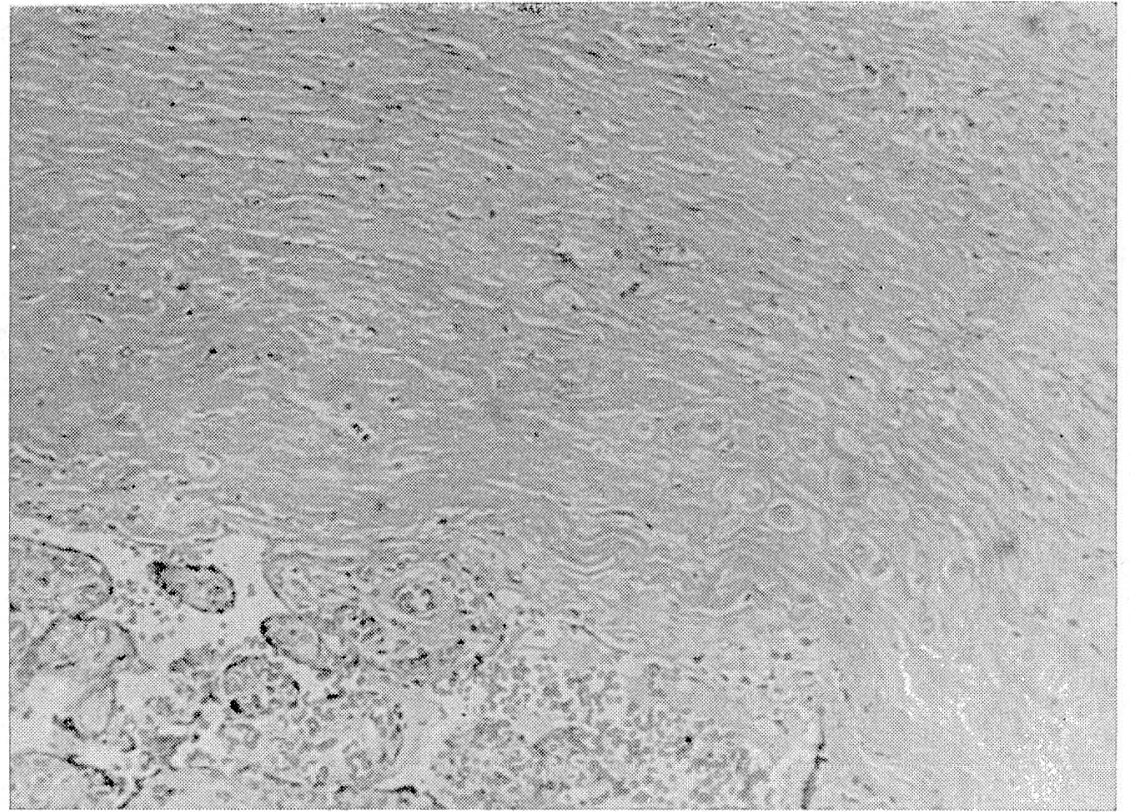

FIGURA No 5 - Microfotografía que muestra la pared de la trompa uterina (a nivel de la fimbria) y las vellosidades placentarias. 


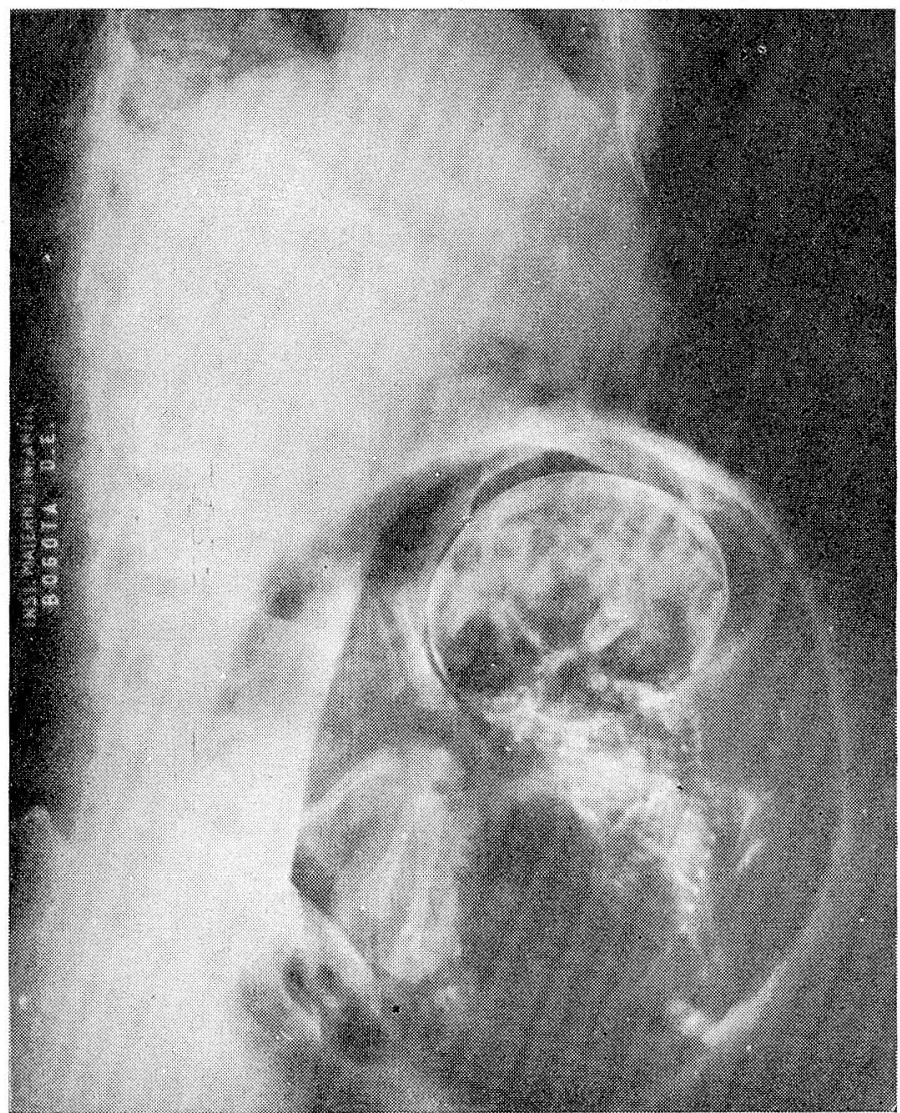

FIGURA No 6 - Los Rx. del tercer caso muestran la falta del contorno uterino, abundante aire en la cavidad amniótica y los signos de muerte fetal.

\section{Diagnóstico}

EMBARAZO FIMBRICO A TERMINO CON FETO FEMENINO DE 2.850 GRS. HIPERMADURO. MACERADO. - SALPINGOOFORECTOMIA DERECHA POR EMBARAZO FIMBRICO. APENDICITIS.

SEGUNDO CASO. H. Clínica $\mathrm{N}$ ? 172560. Mujer de 34 años, (G2. PO. A1.), quien consulta por dolor en hipogastrio y ausencia de movimientos fetales ( 17 IV 68) desde hace 20 días. Aborto hace 6 años. U.M. 2-VIII-67, a los dos meses de embarazo fue tratada por amenaza de aborto. El cuello es duro, largo y cerrado. Se hospitaliza con el diagnóstico de feto muerto y retenido. Los Rx. de abdomen simple comprobaron feto en situación transversa con signos de muerte. Se indujo con pitocín en varias oportunidades y se practicó maniobra de Krauz, sin obtener ninguna respuesta, en vista de lo cual se piensa en embarazo abdominal y se lleva a cirugía y se encuentra masa abdominal adherida a asas intestinales y la placenta localizada en el fondo uterino. Se practica extirpación de la masa y S.O.I. La anatomía patológica (P.Q. No 81568), confirma el diagnóstico de embarazo abdominal secundario con feto femenino, macerado de más o menos 32 semanas, sin ninguna malformación congénita. La placenta muestra severa hialinización y la trompa una salpingitis crónica.

TERCER CASO. H. Clínica No 226990. Mujer de 32 años (G4. P.3) U.M. 11-VII-70), remitida de hospital de provincia (22-V-71), con el diagnóstico de feto muerto 8 días antes, 
donde le habían inducido el parto con pitocín y soluciones hipertónicas, dentro de la cavidad uterina, en varias ocasiones, sin ningún resultado. Al momento de su ingreso se aprecia una paciente en regulares condiciones generales, febril (38\% C.), ligeramente deshidratada, abdómen distendido. Al T.V. cuello blando, entreabierto $y$ se confirma el diagnóstico de feto muerto, palpándose fácilmente a través de pared abdominal. Los Rx. evidencian muerte fetal con abundante aire en cavidad amniótica. (Fig. No 6). Se indujo con pitocín fracasando la inducción. Se sospechó embarazo abdominal practicándosele histerometría, pero el instrumento pasó totalmente. Se interviene con el diagnóstico de feto muerto macerado e infección amniótica; para sorpresa del cirujano, encuentra en cavidad abdominal feto macerado en situación transversa, con el cordón umbilical amputado a $10 \mathrm{cms}$. del ombligo. El útero estaba intacto, ligeramente aumentado de tamaño, friable, anexo izquierdo normal. En el derecho se apreció una masa con múltiples adherencias donde estaba involucrada la placenta y a través de la cual pudo pasar el histerómetro. El feto era de sexo masculino, de 2.400 grs. y no presentaba ninguna malformación congénita. Creemos que se trató de un embarazo tubárico que llegó a término, pero que posibelmente por las amniocentesis repetidas se rompió el saco, apareciendo el feto libre en cavidad abdominal.

\section{Comentario}

Siendo una entidad poco frecuente su incidencia varía, Eisenman y col. (25) dá una frecuencia de $1 \times 15.000$ según Grech y col. (27) es de 1 x 1.814 partos. Entre nosotros la frecuencia es de $1 \times 45.311$ nacidos vivos. Dentro de este lapto de tiempo se encontraron 708 ectópicos y 3 embarazos abdominales a término dándonos un porcentaje del $0.42 \%$ con relación a todos los extrauterinos. Aubert (28) ha observado que la entidad es más frecuente en las razas de color, de 249 mujeres el 90\% eran negras y manifiesta que posiblemente la causa es debida a la falta de diagnóstico y tratamiento del embarazo ectópico en sus primeros estadios, enfatiza sobre la supervivencia que solo es del $10 \%$ y la alta frecuencia de las malformaciones congénitas que pueden ser debidas a la falta de protección de las paredes uterinas y a la escasez del líquido amniótico. En nuestros 3 casos no se presentó ninguna malformación congénita como tampoco sobrevivencia. White (29) obtiene por laparotomía una niña de 2.870 grs. que sobrevive siendo la madre una tuberculosa que hizo T.B.C. genital y la niña no padeció de la enfermedad. Schlussel y col. (30) siguió por 5 años a madre e hijo, al cual extrajo por laparotomía de un embarazo abdominal. Magis y col. (31) de 6 casos reporta 2 sobrevivientes y encuentra una gran incidencia para los Bantues. Aubert (28) también describe un caso, afortunado, de embarazo fímbrico en una mujer de 32 años, cuyo feto de 2.300 grs. aún vive. Clark y col. (32) en su extensa revisión de 18 años en el Freedman's Hospital, presenta 2 casos vivos de los 7 embarazos abdominales a término.

De lo expuesto anteriormente podemos concluir que el embarazo ectópico a término ya sea primario o secundario es un reto para el tocólogo porque es una enfermedad de difícil diagnóstico $(33,34,35$ y 36$)$ que empobrece la sobrevivencia del feto y aún de la madre si no se piensa en él; si se tienen en cuenta la anamnesis, la falta de respuesta a los ocitócicos y la radiografía, el diagnóstico es fácil.

\section{Resumen}

Queremos contribuir a la literatura al aportar 3 casos más de embarazo abdominal a término (con un fímbrico a término); concluímos que es una entidad realmente poco frecuente y de difícil diagnóstico, si no se piensa en ella; cuando se tiene en mente, el diagnóstico se hace sencillo mediante la anamnesis: cirugía previa (cesáreas, miomectomías o 
maniobras abortivas), el fracaso de la respuesta a la inducción con ocitócicos, la histerometría y la radiografía, los cuales son valiosos elementos que confirman el diagnóstico y permiten intervenir oportunamente, disminuyendo así la mortalidad fetal.

\section{Summary}

We wish to contribute to the compilation of literature with 3 cases of abdominal pregnancy at term (with a term fimbria); we have concluded that this is an infrequent condition of difficult diagnosis, if not thought about; when assumed, the diagnosis is simple through anamnesis: previous surgery (cearoctomies) - myomectomies or abortive maneuvers-, the failure of the response to the introduction of oxytocics, hysterectomy and X-ray, which are valuable elements that confirm the diagnosis and enable proper treatment, thus reducing fetal mortality.

\section{BIBLIOGRAFIA}

1 MARTINEZ, P.: Un caso de preñez extrauterina (tubárica) de 13 meses de duración. Rev. Med. Bogotá. Pág. 129 (Julio) 1912.

2 SERPA, R.: El embarazo extrauterino. Rev. Fac. Med. Bogotá, 8: 455, 1940.

3 NAVAS ANGEL, G.: Embarazo ectópico avanzado. Tesis de Grado. 1948.

4 RAMIREZ MERCHAN, R.: Embarazo ectópico casi a término. Relato de 2 historias clínicas. Med. y Cir. Bogotá. 10: 307. 1946.

5 GOMEZ PALACINO, J. A.: Un embarazo tubo-intersticial a término. Rev. del Hospital San Juan de Dios, Bogotá. 1: 101, 1953.

6 GOMEZ PALACINO J. A.: Noventa y cinco casos de embarazo ectópico. Rev. Col. Obst. Ginec. 5: 175, 1954.

7 SANCHEZ TORRES F.: Un caso de embarazo extrauterino avanzado. Rev. Col. Obst. Ginec. 12: 286, 1961.

8 GUZMAN ALANDETE, R.: Embarazo abdo- minal secundaroi a partir de muñón resto tubárico. Rev. Col. Obst. Ginec. 16: 113, 1965.

9 BOTERO URIBE, J.: Embarazo ectópico avanzado. Revisión de la literatura colombiana y presentación de 8 nuevos casos. Rev. Col. Obst. Ginec. 11: 113, 1960.

10 ACOSTA BENDEK, E.: Comentario sobre 2 casos de embarazo ectópico a término. Rev. Col. Obst. Ginec. 8: 195, 1957.

11 LONDOÑO UPEGUI, A.: Una historia de embarazo abdominal. Rev. Col. Obst. Ginec. 5: 206, 1954.

12 GOMEZ PEÑA, J.: Un caso de embarazo tubárico gemelar a término. Rev. Soc. Obst. Ginec. Venezuela. 13: 376, 1953.

13 FRIEDRICH, E. G. et al.: Primary pelvic peritoneal Pregnancy. Obst. Gynec. 31 : 649,1968

14 NEAR, y et al.: A case of hepatic implantation pregnancy with a living infant. Sem. Hosp. Paris. 41: 1430, 1965.

15 HOANG NGOC.: La grossese. A propos d'une observation. (ovarian pregnancy. A Case report). Gynec et Obst. 62: 365, 1963.

16 HUTCHINSON, W. L.: Simultaneus extrauterine and intrauterine pregnancies progressing to viability. Obst. and Gynec. 25: $882,1965$.

17 SEHDER, H. S. et al.: Combined abdominal and intrauterine pregnancies in an African primigravida. Canad. Med. Ass. J. 95 : 1322,1966

18 ACUÑA, G.: Embarazo ectópico avanzado. Rev. Col. Obst. Ginec. 16: 17, 1965.

19 CRAWFORD, J. D. and WORD, J. V.: Advanced abdominal pregnancy, Obst. and Gynec. 10: 549, 1957.

20 BLUTH, I.: Embarazo abdominal. Clin. Obst. Ginec. Pág. 30, (marzo) 1966.

21 RAMIREZ MERCHAN R.: Un caso de embarazo ovárico llegado a término. Revista Médica. 48: 637, 1946.

$22 \mathrm{~K}^{\prime} \mathrm{EY}$ and YENINING C.: Histerosalpingography in the diagnosis of abdominal pregnancy. Clin. Med. J. 83: 218, 1964.

23 HABER, S. Tt. al.: Pelvic angiography in the Management of abdominal pregnancy. Radiology. 85: 904, 1965. 
24 MARTIN, R. E., MATTHEWS, G. B.: Uterine Phebografy in 2 cases of abdominal pregnancy. A. J. Obst. Gynec. 86: 530, 1963.

25 EINSENMAN, J. L. et al.: Pelvic Angiography in retained placenta of abdominal pregnancy. Report of a case. Obst. Gynec. 28: $637,1966$.

26 DILTS, P. J. (Jr.): Pelvic angiography in the diagnosis of abdominal pregnancy. A. J. Obstet. Gynec. 96: 588, 1966.

27 GRECH, P. et al.: The radiological diagnosis of advanced extrauterine pregnancy. Brit. J. Radiol. 38: 848, 1965.

28 AUBERT, W.: On a fortunate case of abdominal pregnancy to term with a living child. Bulletin Fed. Gynec. Obstet. France, 18: $175,1966$.

29 WHITE, A. J.: Abdominal pregnancy, with sur-vival of the child, following genital tuberculosis, J. Obstet. Gynec. Brit. Comm. 73 : 490, 1966.
30 SCHLUSSEL, S., SALL, S.: Advanced abdominal pregnancy: survival with 5 year follow-up. Report of a case. Obstet, and Gynec, 22: 390, 1963.

31 MAGIS. P.: Six cas de grossesse abdominal a terme. Belge Gynec, Obstet, 33: 403, 1963.

32 CLARK, J. F. et al.: Abdominal Pregnancy. A. J. Obstet, Gynec. 96: 511, 1966.

33 YAGI, S. et al.: A case of abdominal pregnancy with a foetus remaining in the abdominal cavity for 15 years. Obstet. and Gynec. (Japan), 14: 231, 1962.

34 FERRARI, B., PAVONE, G., RALFINI G.: La gravidanza abdominale secondaria. Quad. Clin. Obstet. Ginec. 18: 123, 1963.

35 RENGIFO, J. A.: Comentarios sobre gestación ectópico-abdominal y ovárica. Universitas Med. Bogotá, 4: 258, 1959.

36 POKROVSKAYA, N. K.: Full-term tubo-abdominal pregnancy, Akusth 1. Ginek, (ruso). 3: 131, 1963. 